\title{
Comparison of two different strategies for investigating individual differences among consumers in choice experiments. A case study based on preferences for iced coffee in Norway
}

Article

Accepted Version

Creative Commons: Attribution-Noncommercial-No Derivative Works 4.0

Asioli, D., Almli, V. L. and Næs, T. (2016) Comparison of two different strategies for investigating individual differences among consumers in choice experiments. A case study based on preferences for iced coffee in Norway. Food Quality and Preference, 54. 79 - 89. ISSN 0950-3293 doi:

https://doi.org/10.1016/j.foodqual.2016.07.005 Available at https://centaur.reading.ac.uk/75176/

It is advisable to refer to the publisher's version if you intend to cite from the work. See Guidance on citing.

To link to this article DOI: http://dx.doi.org/10.1016/j.foodqual.2016.07.005

Publisher: Elsevier

All outputs in CentAUR are protected by Intellectual Property Rights law, including copyright law. Copyright and IPR is retained by the creators or other copyright holders. Terms and conditions for use of this material are defined in the End User Agreement. 


\section{www.reading.ac.uk/centaur}

\section{CentAUR}

Central Archive at the University of Reading

Reading's research outputs online 


\section{ABSTRACT}

Two different strategies for investigating individual differences among consumers in choice experiments using the Mixed Logit Model are compared. The study is based on a consumer study of iced coffees in Norway. Consumers $(n=102)$ performed a choice task of twenty different iced coffee profiles varying in coffee type, production origin, calorie content and price following an orthogonal design. Consumer attributes, such as socio-demographics, attitudes and habits, were also collected. Choice data were first analysed using the Mixed Logit Model and then two different approaches were adopted for investigating consumer attributes. The first strategy, called one-step strategy, includes the consumer attributes directly in the Mixed Logit Model. The second strategy, called multi-step strategy, combines different methods of analysis such as Mixed Logit Model based on the design factors only, followed by Principal Component Analysis and Partial Least Squares regression to study consumer attributes. The two approaches are compared in terms of data analysis methodologies, outcomes, practical issues, user friendliness, and interpretation. Overall, we think the multistep strategy is the one to be preferred in most practical applications because of its flexibility and stronger exploratory capabilities.

\section{INTRODUCTION}

\subsection{Conjoint Analysis (CA)}

One of the most frequently used methodologies for consumer studies is conjoint analysis (CA). This is a method which is able to estimate the structure of consumer evaluations using a set of product profiles consisting of predetermined combinations of product attributes (Green \& Srinivasan, 1990). Consumers are presented with these product profiles and are asked to either rank, rate or choose among them (Louviere, Hensher, \& Swait, 2000; Molteni \& Troilo, 
2007). Within CA there are two main categories: (i) acceptance-based approaches, which require that consumers rate each alternative product according to their degree of liking or hypothetical purchase intention and (ii) preference-based approaches, where consumers are required to express their preferences either in terms of ranks or of choices among several alternative products with varying levels of attributes. In this paper we will focus on the choice approach.

\subsection{Choice experiment (CE)}

Choice based experiments (CEs) have been developed for investigating consumers' choice both for market and non-market goods (Haaijer, Kamakura, \& Wedel, 2001; Louviere, Hensher, \& Swait, 2000; Yangui, Akaichi, Costa-Font, \& Gil, 2014). In a choice study, consumers are presented with a series of alternative choice scenarios and are asked to choose their most preferred option within each choice scenario. The different alternatives are composed of different combinations of attribute levels which characterize the goods (e.g. price, nutritional content, etc.) usually based on an experimental design. One of the arguments put forward for choice-based methods in comparison to rating or ranking methods, is that having respondents choose a single preferred stimulus among a set of stimuli better approximates a real purchase situation (Carson et al., 1994; Louviere et al., 2000). CEs originate from economics and are increasingly expanding to different fields such as transportation, environment, health and marketing. During the last years there have been an increasing number of applications of CEs also in food consumer studies (Lusk, Fields, \& Prevatt, 2008; Van Loo, Caputo, Nayga, Meullenet, \& Ricke, 2011; Van Wezemael, Caputo, Nayga, Chryssochoidis, \& Verbeke, 2014). 
Consumer heterogeneity with respect to preference pattern, described as "a key and permanent feature of food choice" by Combris, Bazoche, Giraud-Héraud, \& Issanchou (2009), is an important and natural element of food choice research (Almli, Øvrum, Hersleth, Almøy, \& Næs, 2015). Preference heterogeneity can be investigated in terms of demographics (e.g. gender, age, income), attitudes (e.g. preference for certain product characteristics) and habits (e.g. ways and location of food consumption), and is of particular importance for food practitioners (Næs, Brockhoff, \& Tomic, 2010) in order to develop and market food products that better meet consumers' needs and wishes.

At an overall level and independently from data collection and statistical approach, one can identify two main strategies of consumers segmentations: $a$ priori segmentation and $a$ posteriori segmentation (Næs, et al., 2010; Næs, Kubberød, \& Sivertsen, 2001). The a priori segmentation is based on splitting the consumer group into segments according to consumer attributes and then analyzing the group preferences separately or together in an ANOVA model or a Mixed Logit model (depending on data collection, see e.g. Asioli, Næs, Øvrum, \& Almli, 2016) that combine design factors and consumer attributes in one single model (Næs, et al., 2010).

The second strategy is called a posteriori segmentation and is based on creating consumer groups of similar product preferences by analyzing the actual preference, liking or purchase intent data to create segments, and then relating segments to consumer characteristics $a$ posteriori. According to Gustafsson, A., Herrmann, A., \& Huber (2003) there are different approaches to a posteriori segmentation. The main advantage of a posteriori segmentation is that it is unsupervised in the sense that the segments are determined without external influence of consumer attributes, so it is more open to new and unexpected results (Næs, et 

experiment investigating consumer preferences for iced coffee products in Norway were used. al., 2010). In this paper we will use an approach based on visual inspection of scores plots from principal components analysis (PCA) (see e.g. Endrizzi, Gasperi, Rødbotten, \& Næs, 2014), but other possibilities also exist. An important example here is Latent Class Analysis (LCA) which is based on a mathematical optimisiation criterion developed for splitting the group of consumers into segments with similar response pattern (Boxall \& Adamowicz, 2002).

It should be mentioned that there also exists another option more or less between the two segmentation strategies discussed above. This is based on using the consumer attributes explicitly in the segmentation procedure as done in for instance by Vigneau, Endrizzi, \& Qannari (2011). In this paper, however, only a priori and a posteriori segmentation will be in focus.

\subsection{Objectives of the study}

The objective of this study is to compare two different strategies of investigating consumer attributes in CEs, one a priori and one a posteriori strategy. The first strategy includes consumer attributes a priori together with product attributes in a Mixed Logit model and is therefore a one-step strategy. The second strategy is a two-step strategy based on investigating consumers with similar/dissimilar choices using a Mixed Logit model followed by Principal Component Analysis (PCA) and partial least squares (PLS) regression (Wold, Martens, \& Wold, 1983) or PLS classification (Ståhle \& Wold, 1987) for relating the preference pattern to the consumer attributes a posteriori. To compare the methods, data from a conjoint choice Practical issues, user-friendliness and interpretation of the two approaches will be discussed. 


\section{THEORY: STATISTICAL METHODS USED}

120 Choice-based data are routinely analysed within a random utility framework called Discrete

121 Choice Models (DCMs) (Train, 2009). The approach is based on modelling "utility", that is to

122

123

124

125

126

127

128

129

130

131

132

133

134

135

136

137

138

139

140

141

142 say the net benefit a consumer obtains from selecting a specific product in a choice situation, as a function of the conjoint factors. DCMs aim at understanding the behavioural process that leads to a consumer's choice (Train, 2009). DCMs emerged some decades ago and have undergone a rapid development from the original fixed coefficients models such as multinomial logit, to the highly general and flexible Mixed Logit (ML) model. In the ML model, the utility of a product $j$ for individual $m$ in a choice occasion $t$ is written:

$$
\mathrm{U}_{\mathrm{mjt}}=\beta^{\prime} \mathrm{x}_{\mathrm{mjt}}+\varepsilon_{\mathrm{mjt}}
$$

where $\beta_{\mathrm{m}}$ is a random vector of individual-specific parameters accounting for preference heterogeneity, $\mathrm{x}_{\mathrm{mjt}}$ is a vector of conjoint factors, and $\varepsilon_{\mathrm{mjt}}$ is a random error term. For the ML model it is assumed that the random errors are independent identically distributed (i.i.d) and follow a so-called extreme value distribution (see Train, 2009 for theoretical argument for the distributional assumption). An advantage of the ML model is that one may freely include random parameters $\beta_{\mathrm{m}}$ of any distributions and correlations between random factors. This flexibility allows writing models that better match real-world situations. ML models have been applied also in consumer food studies (Alfnes, 2004; Bonnet \& Simioni, 2001;

Hasselbach \& Roosen, 2015; Øvrum, Alfnes, Almli, \& Rickertsen, 2012). In Øvrum et al. (2012) CE was used for investigating how diet choices are affected by exposure to diet-related health information on semi-hard cheese. Hasselbach \& Roosen (2015) investigated whether the concepts of organic and local food support or threaten each other in consumers' choice by using a CE. Alfnes (2004) investigated Norwegians consumers' preferences for country of origin and hormone status of beef using the ML model. In these studies, as in most studies 
143 which apply the ML model, consumers' heterogeneity was not investigated in depth (i.e.

144 segmentation).

146 In the next two sections (2.1 and 2.2), the two strategies introduced in Section 1.3 will be

147 described. attributes (One-step strategy with a priori segmentation)

151 The first strategy is inspired by the analysis of individual acceptance ratings using a Mixed

152 Model ANOVA approach (see e.g. Næs, Almli, Bølling Johansen, \& Hersleth, 2010). It

153 consists of including both conjoint factors and categorical consumer characteristics and their

154 interactions in one model. This means that in addition to the conjoint factor $\mathbf{x}_{\mathbf{m} \mathbf{j}}$ in the model

155 above, one adds additional variables that represent the consumer attributes. In practice, the 156 number of attributes added in this way should be limited due to the lowering of power and

157 also possible more complex interpretation. Note that attributes added in this way could also in

158 principle be based on consumer segments (obtained by for instance an initial analysis) other

159 than those obtained by using the measured consumer attributes individually.

160 Note that interactions between conjoint factors and consumer attributes are of special

161 importance since they represent how the different consumer groups respond differently to the

162 different conjoint factors. This strategy is the same as used in Asioli et al. (2016) for

163 analsying the same data set as used here. 


\subsection{STRATEGY 2: Combining Mixed Logit model, PCA and PLS regression (Multi-step}

\section{strategy with a posteriori segmentation)}

The second strategy has been initially proposed within the framework of Mixed Model ANOVA (Endrizzi et al., 2014; Endrizzi, Menichelli, Johansen, Olsen, \& Næs, 2011; Næs, Almli, et al., 2010). However, this approach can also easily be extended to choice data using the Mixed Logit model (Almli et al., 2015). First, choice data are analyzed using the ML model by including only conjoint factors and possibly also their interactions, as presented in Eq. 1). Then, the matrix of individual parameter estimates $\hat{\boldsymbol{\beta}}_{m}$ extracted from the ML model are analyzed and interpreted using Principal Component Analysis (PCA). At this point, two different approaches for investigating consumer attributes can be applied.

Option 1. A first possible approach is to relate the PCs directly to consumer attributes using for instance Partial Least Squares regression (Endrizzi et al., 2011) which can easily handle a large number of highly collinear attributes. Note that one could also use the parameter estimates $\hat{\boldsymbol{\beta}}_{m}$ directly as responses in the PLS regression or several principal components at the same time. The choice made here of using the PCs as dependent variables was made since the principal components correspond more or less $100 \%$ to the design variables, and since it is of major interest to investigate explicitly how the consumer attributes relate to the different conjoint factors in the design. This option also facilitates the comparison with the first analysis strategy described above (Strategy 1). In order to highlight this aspect, each principal component was handled independently.

Option 2. A second possible approach is to identify segments in the Principal Component Analysis (PCA), either visually (visual segmentation, Endrizzi et al., 2011) or automatically 
189 (using cluster analysis). Then, the consumer segments are investigated in terms of socio-

190 demographics, habits and attitudes attributes using for instance Partial Least Squares -

191 Discrimintion Analysis (PLS-DA, Barker \& Rayens, 2003; Ståhle \& Wold, 1987) which

192 relates the consumer segments to consumer attributes. The main advantage of such an

193 approach is that one can decide during the second step which segments or groups of

194 consumers one is interested in investigating. An application of this method is provided by

195 Almli et al. (2015) who used this approach on ranking data in a consumer study of semi-hard 196 cheese.

197

198 In this paper, all PLS regressions and PLS-DA models were run on standardised input

199 variables, using cross-validation on 10 random segments and performing a jack-knife uncertainty test with $95 \%$ confidence interval for the detection of significant variables

201 (Martens \& Martens, 2000). Calculations were performed in The Unscrambler X 10.2 (Camo

202 Software AS, Oslo). Due to the large number of consumer attributes collected, a two-step

203 procedure was used: in the first step all the consumers' attributes were included in the model.

204 Then, in the second step a new model was run only including significant consumers'attributes 205 from the first step. This results in a better suited and more parsimonial model. For the PLS-

206 DA the consumer groups were represented by dummy variables (Ys) in the PLS-DA, while 207 consumer attributes were used as independent variables (Xs).

\section{MATERIAL AND METHODS}

\subsection{Consumer test}

211 We tested the approaches using a dataset based on iced-coffee products. A sample of 102

212 consumers was recruited in the region south of Oslo, Norway, in November 2012. The test 
213 included four sessions, one of them being a choice task. For details about the experiment and

214 socio - demographic characteristics of the sample investigated, see Asioli et al. (2016).

\subsection{Iced coffee products}

217 Conjont factors and their levels for the iced coffee profiles presented to the consumers were 218 selected based on focus group results; see Asioli, Næs, Granli, \& Lengard Almli (2014) for

219 details. Table 1 shows the four conjont factors and levels that were selected: coffee type, 220 calorie content and origin with two levels each, and price with three levels.

\section{Table 1 - Conjont factors and levels used in the conjoint design}

\subsection{Choice task}

226 An orthogonal choice design composed of eight choice sets of three products each was

227 generated in SAS version 9.3 (see appendix I). The design featured 20 unique samples where 228 all of them were taken from the full factorial design (see Asioli et al, 2016 for more details) .

229 Usually in choice studies a "no-choice" option is included because it can provide a better 230 market penetration prediction (Enneking et al., 2007; Haaijer et al., 2001). However, in this 231 paper we did not aim to predict market penetration, thus we decided not to include the "no232 choice" option and only iced-coffee consumers were recruited to the test. 
234 The eight triads of iced coffee profiles were displayed successively on a computer screen in 235 the form of photographs (see Figure 1).

Figure 1 - One of the iced coffee profiles $<<$ Please, place here figure $1>>$

240 Product presentation was randomized across participants both at choice set level and at 241 product level within choice sets. For each choice-set, consumers' probability of buying was 242 elicited with the question: "Imagine that you are purchasing iced coffee. Which of these iced 243 coffees are you most likely to buy?" and participants answered by clicking on one of the three 244 alternatives.

\subsection{Consumer attributes}

247 In order to investigate individual differences, we have collected a number of consumer

248 attributes. The attributes investigated are related to iced coffee consumption habits

249 (importance of attributes for purchasing, consumption frequency, duration (years) of iced

250 coffee consumption, consumption time of the day, location of consumption, location of 251 purchasing, alternative products, motivations of consumption and types of products), warm 252 coffee habits (types of additives, location of consumption), food attitudes (items of food 253 neophobia, health consciousness and ethnocentricity) and socio-demographic attributes.

254 Consumers attributes are measured using both numerical and categorical variables. For the 255 importance of attributes for choosing iced coffee, the scale is anchored in 1 (Not important at 256 all) and 5 (Very important at all). The same is the case for the habits attributes. All the 
categorical attributes have been coded using dummy variables where 0 represents the absence of the actual level while 1 represents the presence of the attribute level. The complete list of attributes can be obtained from the authors.

\title{
3.5 Data analysis
}

All conjoint factors were coded using effects coding (-1; 1) (Bech \& Gyrd-Hansen, 2005), except price which was coded in three levels (mean centered) $(-1 ; 0 ; 1)$. In other words, the price was coded as a linear covariate (see Asioli et al., 2016 for arguments). For illustration of Strategy 1, we decided to consider only two segmentation attributes, Gender and Age group. Note that many other choices could have been made, these two are only chosen for illustration of the methodology. The factors used were coded as presented in Table 2 .

\section{Table 2 - Factors coded and their description}

\author{
$<<$ Please, place here table 2>>
}

The ML model for the two cases considered here provide both population averages of the regression coefficients and the set of individual coefficients. The population averages can be interpreted directly in terms of p-values and their signs. Magnitudes of the factors can only be considered relative to one another since the utility scale does not represent a true rating scale given by the consumers (see Train, 2009). The standard deviation of the individual coefficients will also be considered in this paper. 


\subsubsection{STRATEGY 1: Simultaneous Mixed Logit model of the conjoint factors and consumer attributes (One-step strategy)}

Following eq. 2) below, we included two consumer attributes in the ML model, namely Gender and Age. Introducing more consumer attributes may make the estimated conjoint effects weaker and thus disturb interpretation (Næs, Almli, et al., 2010); it may also be technically more difficult to achieve in a software context. This is particularly true if there are attributes with several levels or attributes that are continuous. In addition, the attributes may be collinear, making estimation very unstable and the results difficult to interpret. In this paper we confine ourselves to incorporating two consumer attributes Gender and Age.

In our main specification of the model we incoporate main effects of the conjoint factors and all two-factor interactions among the conjoint factors and between the conjoint factors and the consumer attributes. The utility ML model for iced coffee $j$ for individual $i$ in choice occasion $t$ can be written:

$U_{i j t}=\beta_{1 i}$ Coffe $_{i j t}+\beta_{2 i}$ Calories $_{i j t}+\beta_{3 i}$ Origin $_{i j t}+\beta_{4 i}$ Price $_{i j t}+\beta_{5 i}\left(\right.$ Coffee $^{*}$ Calories $_{i j t}+\beta_{6 i}$

$$
(\text { Coffee } * \text { Origin })_{i j t}+\beta_{7 i}(\text { Coffee } * \text { Price })_{i j t}+\beta_{8 i}(\text { Calories } * \text { Origin })_{i j t}+\beta_{9 i}
$$

$$
\left(\text { Calories }^{*} \text { Price }\right)_{i j t}+\beta_{10 i}(\text { Origin } * \text { Price })_{i j t}+\beta_{11 i}(\text { Age } * \text { Coffee })_{i j t}+\beta_{12 i}(\text { Age } * \text { Price })_{i j t}+
$$

$$
\beta_{13 i}(\text { Age } * \text { Calories })_{i j t}+\beta_{14 i}(\text { Age } * \text { Origin })_{i j t}+\beta_{15 i}(\text { Gender } * \text { Coffee })_{i j t}+\beta_{16 i}
$$

$$
(\text { Gender } * \text { Price })_{i j t}+\beta_{17 i}(\text { Gender } * \text { Calories })_{i j t}+\beta_{18}(\text { Gender } * \text { Origin })_{i j t}+\varepsilon_{m j t}
$$

The interaction effects are obtained by multiplying the columns in the data set for the corresponding main effects. The consumer effect is automatically incorporated here since all coefficients are considered random. Note that Gender and Age have no main effect, the reason being that only the relative differences in each individual's utility pattern influences the 
303

304

305

306

choice model. The chosen ML model assumes independent random parameters with normal distributions for all conjoint factors, consumer attributes and two-way interactions. The ML model was estimated using the Stata module mixlogit (Hole, 2007) run in STATA 11.2 software (StataCorp LP, College Station, US). Four thousand Halton draws were used in the simulations. More details on estimation of ML models are found in Train (2009) and Hole (2007). Note that from a segments point of view the interest lies in the interactions between consumer attributes and the conjoint factors. Note also that one can calculate the individual random coefficients and their standard deviations (SDs) for this model as will be shown in Section 4.1.

\subsubsection{STRATEGY 2: Mixed Logit Model, PCA and PLS (Multi-step strategy)}

\section{Mixed Logit Model}

Following eq.1), we developed a Mixed Logit Model which includes the main effects and two-way interactions among conjoint factors. Thus, in our main specification of the model we included all the main effects and interactions among the conjoint factors for Coffee, Calories, Origin and Price. The utility ML model for iced coffee $j$ for individual $i$ in choice occasion $t$ is written:

$$
U_{i j t}=\beta_{1 i} \text { Coffee }_{i j t}+\beta_{2 i} \text { Calories }_{i j t}+\beta_{3 i} \text { Origin }_{i j t}+\beta_{4 i} \text { Price }_{i j t}+B_{5 i}\left(\text { Coffee }^{*} \text { Calories }_{i j t}+\beta_{6 i}\right.
$$

$$
\left(\text { Coffee }^{*} \text { Origin }\right)_{i j t}+\beta_{7 i}(\text { Coffee } * \text { Price })_{i j t}+\beta_{8 i}(\text { Calories } * \text { Origin })_{i j t}+\beta_{9 i}
$$

$$
(\text { Calories } * \text { Price })_{i j t}+\beta_{10 i}(\text { Origin } * \text { Price })_{i j t}+\varepsilon_{m j t}
$$


As can be seen, except for the consumer attributes, the two models are identical. For the technical details on how the calculations have been performed see section 3.5.1.

327 Then, the matrix of individual parameter estimates $\hat{\boldsymbol{\beta}}_{m}$ was extracted from the ML model (Eq.

328 3) by using the command mixlbeta in STATA. Note that this matrix of individual estimates

329 plays a similar role as the residuals matrix from a reduced mixed model ANOVA on rating

330 data in the sense that both reflect individual variations from population effects (Næs, Almli, et 331 al., 2010).

Principal Component Analysis (PCA)

334 The matrix of individual parameter estimates $\hat{\boldsymbol{\beta}}_{m}$ extracted from the Mixed Logit Model analysis is submitted to Principal Component Analysis (PCA) in order to identify the main components of variation between individuals. PCA was conducted in the multivariate statistical software package The Unscrambler X 10.2 (Camo Software AS, Norway).

Partial Least Squares (PLS) regression

340 PLS regression was conducted in the multivariate statistics software package The

341 Unscrambler X 10.2 (Camo Software AS, Norway). Two different ways of relating PCA to 342 consumer attributes will be handled here.

345 In this case the principal components (PCs) are independently related to consumer attributes 346 (here external variables) using simple PLS regression (see Section 2.2 for arguments). 
349 In this case, a visual segmentation based on the first PCA score is performed and used for 350 illustration of the method. Visual segmentation is sometimes more relevant than using a 351 clustering algorithm since there are usually no clear segments in this type of studies (Næs, et 352 al., 2010, Endrizzi et al., 2011). In a visual approach, segmentation can be done according to 353 the interpretation that one is interested in investigating in more detail. Finally, consumers are 354 characterized in terms of socio-demographics, attitudes and habits with the help of a PLS-DA 355 regression model relating the defined segments to the questionnaire.

Note that since this approach is based on the same basic data as for Option 1, one can in many cases not expect large differences in conclusions between the two options. Option 2 is,

359 however, more specific in the sense that it can also be used for segments with a special shape 360 not directly related to one of the components which is the case for the one used below for 361 illustration purposes.

363 We refer to Section 2.2 for a more detatiled analysis of how the PLS regression method was 364 used.

\section{RESULTS} attributes (One-step strategy)

Table 3 contains the estimated parameters of the Mixed Logit model (means and standard deviations) for the main effects of the conjoint factors, their interactions and interactions with

371 sociodemographics terms at population level as well as the variability of the individual 
coefficients as measured by SD. The null hypothesis that all coefficients are zero is rejected by a Wald test ( $\mathrm{p}$-value $<0.001)$ which indicates that the attributes chosen are considered relevant by consumers. The number of observations in the model is equal to 2376 , which corresponds to $\mathrm{n}=99$ participants and not $\mathrm{n}=102$, because three consumers did not declare their age.

Note that the results are slightly different from the results in paper (Asioli et al., 2016) for the same data. The reason for this is that the methods is iterative and that in the present article we used 4,000 so-called halton draws instead of 2,000 in the previous paper (Asioli et al., 2016). As can be seen, however, the p-values for the different tests are quite similar to each other and none of the general conclusions is altered.

Table 3 - Estimated parameters for ML model with conjoint variables' main effects and interactions, and interactions with socio-demographic attributes (Strategy 1). The two columns to the left refer to the population effects while the two columns to the right correspond to the individual differences as measured by standard deviations (SD).

$$
<<\text { Please, place here table 3>> }
$$

On average the consumers prefer low calorie coffees, Norwegian origin and low prices while they do not seem to have any strong differences in preference for the two Coffee types (Table 3). However, Price has a stronger negative effect than Origin and Calories. It is interesting to note that only main effect Coffee type has significant SDs (see Asioli et al., 2016 for more details), indicating large individual differences in preference for this factor. In other words, 
even without a significant overall effect of coffee, there is a lot of individual variation among consumers.

With regard to the interaction effects among conjoint factors the only significant interaction effect (in the population) detected is Coffee*Price $(\mathrm{p}=0.012)$ (Table 3). Thus, consumers who prefer latte are a little bit more sensitive to price changes than consumers who prefer espresso,

400 showing a slightly stronger preference for low price. With regard to the interaction effects crossing conjoint factors with socio-demographic attributes, the most significant interaction that males and females (on average) show different preferences for calorie contents and iced coffee types (i.e. Latte and Espresso). More specifically, females prefer low calories much more strongly than males. Interaction plots illustrating these results are available in Asioli et al. $(2016)^{1}$.

It is interesting to note that there are several interaction effects (i.e. Coffee*Calories, Coffee*Age, Origin*Age, Price*Age) with significant standard deviations (SDs), indicating the relevance of individual differences and also differences within the genders and age groups that are not visible when looking only at the average Gender and Age effects.

4.2 STRATEGY 2: Mixed Logit Model, PCA, PLS regression and PLS discrimination (Multi-step strategy)

\subsubsection{Mixed Logit Model}

416 Table 4 contains the estimated parameters of the Mixed Logit model (means and standard

417 deviations) for the main effects of the conjoint factors and their interactions terms at

\footnotetext{
${ }^{1}$ As indicated before, the model used here is a bit different (different number of iterations), but the results are similar as well as the interaction plots.
} 
population level as well as as the variability of the individual coefficients as measured by SD.

419 Again the null hypothesis that all coefficients are zero is rejected by a Wald test (p-value $<0.01)$.

Table 4 - Estimated parameters for ML model with conjoint variables' main effects and by standard deviations (SD).

From Table 4 we can see again that on average consumers prefer low calories, low prices and Norwegian origin while coffee type is not significant at mean population level which is consistent with results obtained from strategy one (see section 4.1.1). It is interesting to note that all the conjoint factors (main effects) have significant standard deviations (SDs) meaning that there are individual differences in perception. This corresponds to the results in strategy one with significant SD's for several of the interactions with Gender and Age. But as can be seen, in this case without Age and Gender effects, this element appears in the SD's for the main effects themselves. In strategy two these individual differences will be further

436 investigated in the following steps.

437 From Table 4 we can see that only one interaction is significant, namely the interaction 438 between coffee type and price (Coffee*Price), again corresponding to above. 


\subsubsection{Principal Component Analysis (PCA) on regression coefficients}

441 In order to further investigate consumer attributes, a PCA model was run on individual

442 regression coefficient estimates from the ML model above (i.e. model including only main

443 effects and interactions of conjoint factors) (Figure 2). In the PCA model the coefficients are

444 not standardized to preserve the original scale variations. In the following, we concentrate on

445 four principal components (PCs), corresponding very well with the four design factors in the

446 following order: Coffee type (on PC-1, explaining $86 \%$ of the variance), Origin (on PC-2,

447 explaining $6 \%$ of the variance), Calories (on PC-3, explaining $4 \%$ of the variance) and Price

448 (on PC-4, explaining 3\% of the variance). The correspondence between principal components

449 and design factors is natural because of the orthogonality of the design. As can also be seen,

450 the order of importance does not match the relative importance of the factors at a population

451 level (averages) indicated in the ML model, while it corresponds very well with the order

452 indicated by the significant SD's in Table 4. Thus, it is clear that Coffee type explains the

453 largest variance, followed by Origin and Calories. It is also interesting to note that Price

454 contributes least to the variance. This is because there is a strong agreement between

455 consumers in the direction of preferring a lower price for the same product attributes. On the

456 contrary, there is no preferred type of coffee at population level (this main effect is non

457 significant), but a lot of individual variations revealed by the SDs and the PCA results. This

458 clearly shows the shortcomings of only looking at average effects that is often done in many

459 conjoint studies.

460 It is important to emphasize that instead of the PCs of the regression coefficients one could in

461 this case, based on an orthogonal design, have used the main effect estimates for the

462 consumers directly as response variables. For non-orthogonal designs, the relation between

463 main effects and the PCA plot may be more complicated. Using the PCA also opens up the

464 possibility of identifying more easily consumers with for instance large values on two or more 
465

466

467

468

469

470

471

472

473

474

475

476

477

478

479

480

481

482

483

484

485

486

487

of the components. This latter aspect could be important for segmentation purposes as is the case for the Option 2 below.

Figure 2 - PCA correlation loadings plot - for PC-1 and PC-2 - on individual Mixed Logit parameter estimates from choice data (scores are presented in Figure 6) $<<$ Please, place here figure $2>>$

Note: the names placed in the figure on the extremes of PC-1 (Espresso and Latte) and PC-2 (Italy and Norway) have been inserted for a better interpretation of the bi-plot.

\subsubsection{Investigation of consumer attributes}

As indicated in the section 3.5.2 two options for investigating consumer attributes starting from the PCA analysis will be tested. The first option relates consumer attributes as external variables directly to the PCs indentified using for instance PLS regression, while in the second option the consumer attributes are related to segments determined in the PCA plot, using PLS-DA. In all cases, the PLS regression allows for many collinear explanatory attributes which is a clear advantage of the method. The values of the explained variances indicated in the next steps refer to the plots with only significant consumer attributes.

\section{OPTION 1: Relating PCs to consumer attributes}

We applied PLS regression by relating the PCs identified in the PCA above directly to consumer attributes. Due to the independence of the axes, it is most natural here to consider the axes separately (individual PCs), but a joint analysis is also possible (see above). The results from components 3 and 4 will only be mentioned briefly without Figures. 
Figure 3 presents PC-1 (Coffee type) and its relation to consumer attributes. The crossvalidation $(\mathrm{CV})$ indicates that one component is clearly significant, but component two also added slightly to prediction ability. The explained variances for components 1 and 2 are equal to $20 \%$ and $11 \%$ for $\mathrm{X}$ and $50 \%$ and $5 \%$ for $\mathrm{Y}$. We can notice that there is a large number of significant, as determined by the jack-knife method described above for 1 component,consumer attributes as compared to the other PCs (see for instance Figures 4 for 494 PC-2 results). In particular, PC-1 (describing conjoint factor Coffe type, see Figure 3) is positively correlated to espresso coffee habits (preference for high coffee intensity, warm coffee, espresso, americano, regular and black coffee) and males while it is negatively correlated to 498 consumption habits of warm coffee with milk (e.g. milk content, latte and cappuccino) (Table 5). Thus PC-1 describes two directions of coffee type habits, which also indicates the possibility to identify two groups of consumers as we will see in the option two. As can be

501 seen, there is a natural correspondence between the preference pattern and what the 502 consumers indicate that they do/like. The position of the consumer attributes in the plots 503 before and after the significant test is more or less the same in both configurations.

504 Gender Figure 3 - Correlation loadings - PLS components 1 and 2 - with significant consumer attributes from PLS regression model using PC-1 as dependent variable (Coffee type) 
510 Using two components in the significance tests changed the number of significant attributes

511 slightly. In particular, two attributes related to iced coffee habits (preference for brand B and 512 canteen as location of iced coffee consumption) have now a significantly positive correlation

513 to PC-1 (Coffe type direction). On the other hand preference for Brand A iced coffee,

514 americano warm coffee and indication of work/university as usual location of warm coffee

515 consumption are no longer significant. All attributes that are significant for both one and two

516 components PLS models are located in the same positions in both plots. For two components

517 Gender was not significant, but this is not so surprising since Gender is only borderline

518 significant in Strategy 1.

Table 5 - Significant consumers attributes for the one-component model (PC1) (p-values on regression coefficients, from jack-knife test)

$<<$ Please, place here table 5>>

For PC-2, the predictive CV indicated that none of the components was significant, but based on one component the jack-knife significance test gave a number of significant attributes.

526 Figure 4 shows the relation of PC-2 (describing conjoint factor Origin, see Figure 4) with significant consumer attributes. The explained variances for 1 and 2 components are now $36 \%$ and $16 \%$ for $\mathrm{X}$ and $21 \%$ and $1 \%$ for $\mathrm{Y}$. 
534 We can see that PC-2 is positively related to location of iced coffee consumption (i.e.

535 café/restaurant and bar) which is negatively correlated to consumer attributes importance of 536 origin and preference for foods of Norwegian origin and for familiar foods (Table 6). Neither 537 Age nor Gender were significant in this case, which corresponds to the findings from Strategy

538 1. The position of the consumer attributes in the plots before and after the significant test and 539 variable selection is more or less the same.

Table 6 - Significant consumers' attributes for the two-component model (PC1-PC2) (pvalues on regression coefficients, from jack$<<$ Please, place here table $6>>$

For PC-3 (describing conjoint factor Calories) the cross-validation (CV) indicates a slight significance of the first component and therefore only one component was used in the jack-

547 knife test. PC-3 was found to be positively correlated with price and Gender (males) and 548 negatively correlated with calories, use of sweetener and warm coffee habits (i.e. cappuccino 549 and americano). Gender was in this case one of the significant attributes which is positively 550 correlated to PC-3. This indicates that the differences between the calorie levels is more 551 important for the females than it is for the males (Asioli et al., 2016), which is in 552 correspondance with the results for Strategy 1. The position of the consumer attributes in the 553 plots before and after the significant test is more less the same. Finally, PC-4 (which is related 554 to individual differences in perception of price) is positively correlated to origin and 555 negatively correlated to price and calories. Again no component was significant in the cross556 validation, and only one component was used in the jack-knife test. The attributes reported 
557 here are the ones found to be significant. In this case neither Age nor Gender was significant.

558 The position of the consumer attributes in the plots before and after the significance test and

559 variable selection is more or less the same.

560 As we have seen, in these analyses, Gender shows up as significant for PC-1 and PC-3 (i.e.

561 for coffee and calories). This means that the two genders have a different preference for the

562 two coffee types and calories levels, i.e. there is an interaction between the two. This

563 corresponds exactly to what was found in Strategy 1 where the interaction between Gender

564 and the two conjoint factors (coffee type and calories) were the only two interactions found to

565 be significant (see Table 3). In the present Strategy (option one), however, one can also obtain

566 information about the other attributes and how they relate to the conjoint factors which is

567 clearly more difficult in Strategy 1.

568 Quantification of the individual differences in the interactions between Gender and conjoint

569 factors which was a major issue in the previous strategy is, however, less obvious in the

570 present case. One can see clear individual differences in the scores plot regarding preferences

571 along the different conjoint factors, but a numerical statement of significance is not available

572 here, in contrast to Strategy 1.

573 Note that for none of the analyses the significance tests and elimination of the non-signifiant

574 variables changed the general structure/position of the reminaing variables. The elimination of

575 variables must here therefore mainly be considered a way of making plots interpretation

576 simpler.

OPTION 2: Preference heterogeneity and consumer segmentation

Espresso and Latte segments (PCA) 
580 For comparison with the above and for illustrating this second option we decided to

581 concentrate on two equally-sized segments determined along the first PCA axis. It should,

582 however, be emphasised that other PCs can be used to define segments depending on the

583 objective of the study. For example, four segments defined along PC1 and PC2 could also be

584 used as has been done in a previous paper with rating data (Asioli et al., 2014). Indeed, visual

585 segmentation can easily be performed and it is flexible (Almli et al., 2015; Næs, et al., 2010).

586 The consumer segments consist of 51 subjects for the Espresso group and 51 subjects for the

587 Latte group (Figure 5). In the following sections these segments are referred to as "Espresso" 588 and "Latte" segments, respectively (see section 4.2.2).

Figure 5 - PCA scores plot on individual Mixed Logit parameter estimates from choice data

\section{Segments characteristics}

595 To describe the consumer segments in terms of habits, attitudes and socio-demographic

596 attributes an approach based on PLS-DA was used (Figure 6). The consumer groups (Latte 597 and Espresso) were represented by dummy variables (Ys) in the PLS-DA, while consumer 598 attributes were used as independent variables (Xs). The cross-validation (CV) indicates that 599 only one component had a significant prediction ability and therefore only one component 600 was used in the jack-knife test. The explained variances for the first two components were $60129 \%$ and $19 \%$ for $X$ and $34 \%$ and $1 \%$ for Y. Socio-demographic attributes were not found to 602 be significant.With regard to warm coffee consumption habits, the two segments differ 603 significantly for several attributes. Consumers in the Espresso group show the highest 
604 consumption of "Espresso" warm coffee type and also preference for "black" warm coffee.

605 Finally, consumers belonging to the Latte segment have preference for two types of warm

606 coffee: latte and capuccino. These findings are fully coherent with the definition of the two

607 groups. Further, only one iced coffee habit has been found significant which is the preference

608 for Espresso segment of the "B" brand.

609 As can be seen these results are similar to the results of the PC-1 in the option one which is

610 natural since we segmented the consumers based on PC-1. The main reason for incorporating

611 the Option 2 here, however, is that it can also be used for other segments with shapes and

612 positions not directly related to one of the components as was the case here.

613 As can be seen Gender is no longer significant at the fixed significance level. As discussed

614 above this is not totally surprising since Gender is borderline significant and therefore two

615 different tests may lead to different conclusions relative to a fixed significance threshold.

616

617 Figure 6 - Correlation loadings with significant consumer attributes from PLS-DA

618 model

619

$<<$ Please, place here figure 6>>

620

621 5. DISCUSSION

622 The main aim of this paper was to compare two different strategies for investigating

623 individual differences among consumers using choice data collected in a study about

624 consumer preferences for iced coffee products in Norway. The focus of the paper is on

625 methodology and advantages and disadvantages from a methodological point of view. It must, 
626 however, be emphasized that the methods should be compared on more data sets in order to

627 come with more general statements about their properties.

628

\subsection{Comparison of the two strategies in terms of flexibility}

630 The multi-step Strategy (here Strategy 2) can be considered more flexible compared to the 631 one-step Strategy (here Strategy 1) since the latter is only able to investigate a limitated 632 number of pre-defined consumer attributes at a time. The multi-step Strategy on the other 633 hand can be used to investigate a large number of potentially collinear consumer attributes at 634 the same time. This is important since no selection of attributes is needed before analysis. 635 Options 1 and 2 for Strategy 2 are more or less equally flexible. For the first one, one can 636 relate the regression coefficients or their PCs as done here directly to the consumer attributes, 637 while for option 2 one can look at different segments depending on the scope of the analysis. 638 The latter then opens up for a more focused analysis related to what one is most interetested 639 in studying.

\subsection{Comparison of the two strategies in terms of data analysis, computation and} interpretation

643 Data analysis and computation of the one-step strategy can be considered simpler to perform

644 compared to the multi-step strategy. First of all the one-step strategy requires skills and 645 expertise related to only one statistical model (Mixed Logit Model) while in the multi-step 646 strategy three models have to be performed (Mixed Logit Model, PCA and PLS regression). 647 This also means that it may require expertise and skills about two software programs, such as 648 (in this case) STATA 11.2 and The Unscrambler X 10.2. 
649 For the comparison of options 1 and 2 for Strategy 2, the second one is more complex since

650 an additional step of choosing the segments comes in on top of ML modelling and regression.

651 From an interpretation point of view, Strategy 1 is slightly simpler since all results are to be 652 found in one table only. However Strategy 2 has the advantage of using maps which are very 653 easy to understand in comparison with estimate values, especially for non statisticians.

\subsection{Comparison of the two approaches in terms of outcomes}

656 A possible drawback with Strategy 2 is that it is harder to obtain quantitative information 657 about the individual differences in consumers' liking for a conjoint factor within for instance 658 a consumer attribute such as Gender or Age. It may be visible in the plot that such a tendency 659 is clear, but a quantitiave assessment is more difficult to get.

660 For the elements that can be compared the two strategies led in this case to similar results 661 regarding the main and interaction effects among the conjoint factors. Indeed, both strategies 662 show that consumers have strong preferences for low calories, Norwegian origin and low 663 price iced coffee products as main effects, while there is a significant effect for the interaction 664 Coffee*Price. Strategy 2, however, added information about a number of other consumer 665 attributes which may be very important for product development practices.

\section{CONCLUSIONS}

668 This study compared two different ways investigating individual differences and their relation 669 to consumer attributes using choice data. One of the strategies is a one-step a priori 670 segmentation strategy based on joint Mixed Logit modelling of all data. The other strategy is 671 a multi-step strategy based on relating the individual preference results from the Mixed Logit 
672 model to the external consumer attributes by regression or classification methods. Outcomes

673 showed that the two strategies for the actual data gave similar results about main and

674 interaction effects among conjoint factors. For the individual differences, the results were also

675 comparable for the consumer attributes that were considerd in both strategies. The multi-step

676 strategy has the advantage that it is more flexible and can be used to analyse several, possibly

677 collinear, consumer attributes at the same time. An advantage of the one-step strategy is that it

678 gives simpler numerical assessments of individual differences in their assessments of the

679 different conjoint factors. On the other hand, it only allows to focus on few pre-selected

680 consumer attributes. Overall, we think the multi-step strategy is the one to be preferred in

681 most practical applications because of its flexibility and stronger exploratory capabilities.

682 Comparisons of the two methodologies for other data sets are needed in order to evaluate the

683 general validity of the conclusions.

684

\section{ACKNOWLEDGEMENTS}

686 The research was conducted in Norway in the framework of the FOODCHOICE project, a

687 user-driven project funded by the Research Council of Norway focusing on method

688 development within consumer research. Financial support from the Research Council of

689 Norway for the YGGDRASIL mobility program 2012-2013: Project Number 219787/F11 is

690 acknowledged. Thanks also to the European Commission through the Marie Curie Actions

691 Intra European Fellowship (IEF), call FP/-PEOPLE-I2012-IEF - project title “'Innovative

692 Methodologies for New Food Product Development: combining Sensory Science and

693 Experimental Economics - NEFOMET" for the support. Kjell Merok (Nofima) is thanked for

694 designing the iced coffee products profiles.

695 


\section{REFERENCES}

697

698

699

700

701

702

703

704

705

706

707

708

709

710

711

712

713

714

715

716

717

718

Alfnes, F. (2004). Stated preferences for imported and hormone-treated beef: application of a mixed logit model. European Review of Agricultural Economics , 31 (1 ), 19-37.

Almli, V. L., Øvrum, A., Hersleth, M., Almøy, T., \& Næs, T. (2015). Investigating individual preferences in rating and ranking conjoint experiments. A case study on semi-hard cheese. Food Quality and Preference, 39, 28-39.

Asioli, Næs, T., Øvrum, A., \& Almli, V. L. (2016). Comparison of rating-based and choicebased conjoint analysis models. A case study based on preferences for iced coffee in Norway. Food Quality and Preference, 48, 174-184.

Asioli, D., Næs, T., Granli, B. S., \& Lengard Almli, V. (2014). Consumer preferences for iced coffee determined by conjoint analysis: an exploratory study with Norwegian consumers. International Journal of Food Science \& Technology, 49(6), 1565-1571.

Barker, M., \& Rayens, W. (2003). Partial least squares for discrimination. Journal of Chemometrics, 17(3), 166-173.

Bech, M., \& Gyrd-Hansen, D. (2005). Effects coding in discrete choice experiments. Health Economics, 14(10), 1079-1083.

Bonnet, C., \& Simioni, M. (2001). Assessing consumer response to Protected Designation of Origin labelling: a mixed multinomial logit approach. European Review of Agricultural Economics , 28 (4), 433-449.

Boxall, P. C., \& Adamowicz, V. (2002). Understanding Heterogeneous Preferences in Random Utility Models: A Latent Class Approach. Environmental and Resource Economics, 23(4), 421-446.

Carson, R., Louviere, J., Anderson, D., Arabie, P., Bunch, D., Hensher, D., ... Wiley, J. 
Combris, P., Bazoche, P., Giraud-Héraud, E., \& Issanchou, S. (2009). Food choices: What do we learn from combining sensory and economic experiments? Food Quality and Preference, 20(8), 550-557.

Endrizzi, I., Gasperi, F., Rødbotten, M., \& Næs, T. (2014). Interpretation, validation and segmentation of preference mapping models. Food Quality and Preference, 32, 198-209.

Endrizzi, I., Menichelli, E., Johansen, S. B., Olsen, N. V., \& Næs, T. (2011). Handling of individual differences in rating-based conjoint analysis. Food Quality and Preference, 22(3), 241-254.

Enneking, U., Neumann, C., \& Henneberg, S. (2007). How important intrinsic and extrinsic product attributes affect purchase decision. Food Quality and Preference, 18(1), 133138.

Green, P. ., \& Srinivasan, V. (1990). Conjoint Analysis in Marketing: New Developments with Implications for Research and Practice. Journal of Marketing, 54(4), 3-19.

Gustafsson, A., Herrmann, A., \& Huber, F. (2003). Conjoint measurement: Methods and applications. Berlin: Springer.

Haaijer, R., Kamakura, W. A., \& Wedel, M. (2001). The "No-Choice" Alternative in Conjoint Choice Experiments. International Journal of Market Research, 43(1), 93-106.

Hole, A. R. (2007). Fitting mixed logit models by using maximum simulated likelihood. The Stata Journal, 7(3), 188-401.

Johanna Lena, H., \& Roosen, J. (2015). Consumer Heterogeneity in the Willingness to Pay for Local and Organic Food. Journal of Food Products Marketing, 21(6), 608-625.

Louviere, J. J., Hensher, D., \& Swait, J. (2000). Stated choice methods: analysis and 
application. Cambridge.

743

744

745

746

747

748

749

750

751

752

753

754

755

756

757

758

759

760

761

762

763

Louviere, J. J., Hensher, D. A., \& Swait, J. D. (2000). Stated choice methods. Analysis and applications. Cambridge University Press.

Lusk, J. L., Fields, D., \& Prevatt, W. (2008). An Incentive Compatible Conjoint Ranking Mechanism . American Journal of Agricultural Economics , 90 (2 ), 487-498.

Martens, H., \& Martens, M. (2000). Modified Jack-knife estimation of parameter uncertainty in bilinear modelling by partial least squares regression (PLSR). Food Quality and Preference, 11(1-2), 5-16.

Molteni, L., \& Troilo, G. (2007). Ricerche di Marketing. Milano: McGraw-Hill.

Næs, Brockhoff, P., \& Tomic, O. (2010). Statistics for sensory and consumer science.

Næs, T., Almli, V. L., Bølling Johansen, S., \& Hersleth, M. (2010). Alternative methods for combining design variables and consumer preference with information about attitudes and demographics in conjoint analysis. Food Quality and Preference, 21(4), 368-378.

Næs, T., Kubberød, E., \& Sivertsen, H. (2001). Identifying and interpreting market segments using conjoint analysis. Food Quality and Preference, 12(2), 133-143.

Ståhle, L., \& Wold, S. (1987). Partial least squares analysis with cross-validation for the twoclass problem: A Monte Carlo study. Journal of Chemometrics, 1(3), 185-196.

Train, K. (2009). Discrete choice methods with simulation. (C. U. Press, Ed.)New York (Vol. 47). Cambridge University Press.

Van Loo, E. J., Caputo, V., Nayga, R. M., Meullenet, J.-F., \& Ricke, S. C. (2011). Consumers' willingness to pay for organic chicken breast: Evidence from choice experiment. Food Quality and Preference, 22(7), 603-613.

764 Van Wezemael, L., Caputo, V., Nayga, R. M., Chryssochoidis, G., \& Verbeke, W. (2014). 
European consumer preferences for beef with nutrition and health claims: A multicountry investigation using discrete choice experiments. Food Policy, 44, 167-176.

767 Vigneau, E., Endrizzi, I., \& Qannari, E. M. (2011). Finding and explaining clusters of consumers using the CLV approach. Food Quality and Preference, 22(8), 705-713.

Wold, S., Martens, H., \& Wold, H. (1983). Matrix Pencils: Proceedings of a Conference Held at Pite Havsbad, Sweden, March 22--24, 1982. In B. Kågström \& A. Ruhe (Eds.), (pp. 286-293). Berlin, Heidelberg: Springer Berlin Heidelberg.

772 Yangui, A., Akaichi, F., Costa-Font, M., \& Gil, J. M. (2014). Are ranking preferences Healthier Societies” (p. 13). Ljubljana, Slovenia. choices: Results from a cheese experiment. Food Policy, 37(5), 520-529. 


\section{Appendix I - Choice design}

788 "Please, place here appendix I" 


\section{$790 \quad$ Highlights}

791 - Two strategies investigating individual differences using choice data are compared.

792 - Strategy 1 includes the consumer attributes directly in the Mixed Logit Model.

793 - Strategy 2 combines different methods such as Mixed Logit Model, PCA and PLS.

794 - Strategy 2 is preferred for its flexibility and stronger exploratory capabilities.

795 
Table 1 - Conjont factors and levels used in the conjoint design

\begin{tabular}{lll}
\hline FACTORS & LEVELS \\
\hline Coffee type & 1 & Latte \\
& 2 & Espresso \\
Calories & 1 & $60 \mathrm{kcal} / 100 \mathrm{ml}$ \\
& 2 & $90 \mathrm{kcal} / 100 \mathrm{ml}$ \\
Origin & 1 & Norway \\
& 2 & Italy \\
Price & 1 & 17 NOK $(\approx € 2.0)$ \\
& 2 & 23 NOK $(\approx € 2.7)$ \\
& 3 & 29 NOK $(\approx € 3.4)$ \\
\hline
\end{tabular}

797

798

799

800

801

802

803

804

805

806

807

808

809

810

811

812

813

814

815

816

817 
Table 2 - Factors coded and their description

\begin{tabular}{|c|c|}
\hline FACTOR & DESCRIPTION \\
\hline Coffee & If Espresso: 1 ; otherwise (Latte): -1 \\
\hline Calories & If $90 \mathrm{kcal} / 100 \mathrm{ml}: 1 ;$ otherwise $(60 \mathrm{kcal} / 100 \mathrm{ml}):-1$ \\
\hline Origin & If Italy: 1 ; otherwise (Norway): -1 \\
\hline Price & If 17 NOK: $-1 ;$ if 23 NOK: $0 ;$ if 29 NOK: 1 \\
\hline Gender & If Male: $1 ;$ otherwise (Female): -1 \\
\hline Age & If age is $37-56: 1 ;$ otherwise $21-36$ (younger): -1 \\
\hline
\end{tabular}

819

820

821

822

823

824

825

826

827

828

829

830

831

832

833

834

835

836

837

838

839

840

841 
842 Table 3 - Estimated parameters for ML model with conjoint variables' main effects and

843 interactions, and interactions with socio-demographic attributes. The two columns to the

844 left refer to the population effects while the two columns to the right correspond to the

845 individual differences as measured by standard deviations (SD).

\begin{tabular}{|c|c|c|c|c|}
\hline \multirow[t]{2}{*}{ EFFECTS } & \multicolumn{2}{|c|}{ GROUP AVERAGE } & \multicolumn{2}{|c|}{ INDIVIDUAL VARIATION } \\
\hline & Estimate & p-Value & Std. Dev & p-Value \\
\hline \multicolumn{5}{|l|}{ Main effects } \\
\hline Coffee & -0.046 & 0.883 & 2.463 & $0.000 * * *$ \\
\hline Calories & -0.657 & $0.000 * * *$ & 0.317 & 0.232 \\
\hline Origin & -0.500 & $0.005^{* * *}$ & 0.152 & 0.468 \\
\hline Price & -1.696 & $0.000 * * *$ & 0.181 & 0.462 \\
\hline \multicolumn{5}{|c|}{ Interactions among conjoint factors } \\
\hline Coffee*Calories & -0.046 & 0.737 & 0.526 & $0.005 * *$ \\
\hline Coffee*Origin & 0.298 & 0.093 & 0.477 & 0.051 \\
\hline Coffee*Price & 0.316 & $0.012 *$ & 0.008 & 0.947 \\
\hline Calories*Origin & 0.085 & 0.526 & 0.007 & 0.962 \\
\hline Calories*Price & -0.016 & 0.907 & 0.268 & 0.274 \\
\hline Origin*Price & -0.113 & 0.454 & 0.276 & 0.237 \\
\hline \multicolumn{5}{|c|}{ Interactions with sociodemographics attributes } \\
\hline Coffee*Gender & 0.569 & $0.034 *$ & 0.918 & 0.063 \\
\hline Coffee*Age & -0.492 & 0.057 & 1.310 & $0.001 * *$ \\
\hline
\end{tabular}




\begin{tabular}{|l|r|r|r|r|}
\hline Calories*Gender & 0.544 & $0.000^{* * *}$ & 0.105 & 0.648 \\
\hline Calories*Age & -0.144 & 0.258 & 0.660 & $0.001^{*}$ \\
\hline Origin*Gender & 0.075 & 0.661 & 0.281 & 0.170 \\
\hline Origin*Age & 0.144 & 0.391 & 0.136 & $0.000^{* * *}$ \\
\hline Price*Gender & -0.127 & 0.467 & 0.510 & $0.047^{*}$ \\
\hline Price*Age & & & & 0.991 \\
\end{tabular}

${ }^{*},{ }^{* *}$ and ${ }^{* * *}$ indicate significant effects at $0.05,0.01$ and 0.001 levels, respectively. 
Table 4 - Estimated parameters for ML model with conjoint variables' main effects and

870 interactions. The two columns to the left refer to the population effects while the two

871 columns to the right correspond to the individual differences as measured by standard

872 deviations (SD).

\begin{tabular}{|c|c|c|c|c|}
\hline \multirow[t]{2}{*}{ EFFECTS } & \multicolumn{2}{|c|}{ GROUP AVERAGE } & \multicolumn{2}{|c|}{ INDIVIDUAL VARIATION } \\
\hline & Estimate & p-Value & Std. Dev & p-Value \\
\hline \multicolumn{5}{|l|}{ Main effects } \\
\hline Coffee & -0.183 & 0.379 & 1.881 & $0.000 * * *$ \\
\hline Calories & -0.571 & $0.000^{* * * *}$ & 0.557 & $0.000 * * *$ \\
\hline Origin & -0.281 & $0.007 * *$ & 0.666 & $0.000 * * *$ \\
\hline Price & -1.06 & $0.000^{* * * *}$ & 0.596 & $0.000 * * *$ \\
\hline \multicolumn{5}{|c|}{ Interactions among conjoint attributes } \\
\hline Coffee*Calories & 0.061 & 0.537 & 0.204 & 0.393 \\
\hline Coffee* Origin & 0.162 & 0.203 & 0.306 & 0.235 \\
\hline Coffee*Price & 0.229 & $0.015^{*}$ & 0.007 & 0.949 \\
\hline Calories*Origin & 0.046 & 0.676 & 0.042 & 0.711 \\
\hline Calories*Price & -0.062 & 0.500 & 0.073 & 0.752 \\
\hline Origin*Price & -0.111 & 0.335 & 0.052 & 0.763 \\
\hline
\end{tabular}

873

${ }^{* * *}$ and ${ }^{* * *}$ indicate significant effects at $0.05,0.01$ and 0.001 levels, respectively.

874 Number of choice observations: 2448

875 Number of consumers: 102

876

877

878 

on regression coefficients, from jack-knife test)

\begin{tabular}{|c|c|}
\hline CONSUMERS ATTRIBUTES & P-VALUES \\
\hline Coffee intensity & 0.000 \\
\hline Warm Coffee & 0.001 \\
\hline Tine IC & 0.038 \\
\hline Regular C & 0.000 \\
\hline Latte C & 0.000 \\
\hline Espresso C & 0.000 \\
\hline Capp. C & 0.020 \\
\hline Mocca C & 0.015 \\
\hline Americano $\mathrm{C}$ & 0.017 \\
\hline Black & 0.000 \\
\hline Milk & 0.001 \\
\hline Work/Un C & 0.019 \\
\hline Gender & 0.040 \\
\hline
\end{tabular}

881

882

883

884

885

886

887

888

889

890

891

892

893

894

895

896 
897 898

899

900

901

902

903

904

905

906

907

908

909

910

911

912 values on regression coefficients, from jack-knife test)

\begin{tabular}{|c|c|}
\hline CONSUMERS ATTRIBUTES & P-VALUES \\
\hline Origin & 0.027 \\
\hline Late at night & 0.049 \\
\hline Café'/restaurant & 0.029 \\
\hline Bar IC & 0.026 \\
\hline Best food own & 0.000 \\
\hline Stick foods & 0.002 \\
\hline Norwegians & 0.000 \\
\hline
\end{tabular}

Table 6 - Significant consumers' attributes for the two-component model (PC1-PC2) (p-

(1) 
Appendix I - Choice design

\begin{tabular}{|c|c|c|c|c|}
\hline SET & COFFEE TYPE & $\begin{array}{c}\text { CALORIES } \\
\text { (kcal per } 100 \mathrm{ml} \text { ) }\end{array}$ & ORIGIN & $\begin{array}{l}\text { PRICE } \\
\text { (NOK) }\end{array}$ \\
\hline \multirow{3}{*}{1} & Espresso & 90 & Italy & 23 \\
\hline & Latte & 60 & Norway & 17 \\
\hline & Latte & 90 & Norway & 29 \\
\hline \multirow{3}{*}{2} & Latte & 90 & Italy & 29 \\
\hline & Latte & 90 & Italy & 17 \\
\hline & Espresso & 60 & Norway & 23 \\
\hline \multirow{3}{*}{3} & Espresso & 60 & Norway & 29 \\
\hline & Latte & 60 & Italy & 17 \\
\hline & Latte & 90 & Norway & 23 \\
\hline \multirow{3}{*}{4} & Espresso & 90 & Norway & 29 \\
\hline & Espresso & 60 & Italy & 23 \\
\hline & Latte & 60 & Italy & 17 \\
\hline \multirow{3}{*}{5} & Espresso & 60 & Norway & 17 \\
\hline & Latte & 60 & Italy & 29 \\
\hline & Latte & 90 & Italy & 23 \\
\hline \multirow{3}{*}{6} & Latte & 60 & Norway & 29 \\
\hline & Espresso & 90 & Norway & 17 \\
\hline & Espresso & 60 & Italy & 23 \\
\hline 7 & Latte & 90 & Norway & 23 \\
\hline
\end{tabular}




\begin{tabular}{|c|c|c|c|c|}
\hline \multirow{4}{*}{} & Espresso & 90 & Italy & 17 \\
\cline { 2 - 5 } & Espresso & 60 & Italy & 29 \\
\hline \multirow{3}{*}{8} & Latte & 60 & Norway & 29 \\
\cline { 2 - 5 } & Espresso & 90 & Italy & 17 \\
\hline
\end{tabular}

914

915 
$917 \quad$ Figure 1 - One of the iced coffee profiles

918 


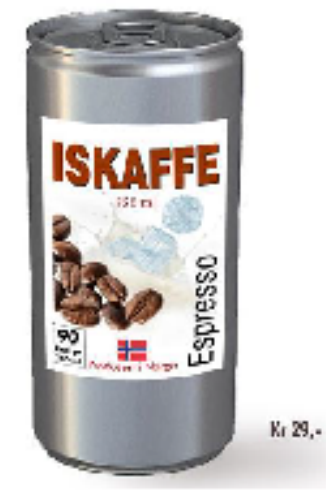

Figure 1 - One of the iced coffee profiles 


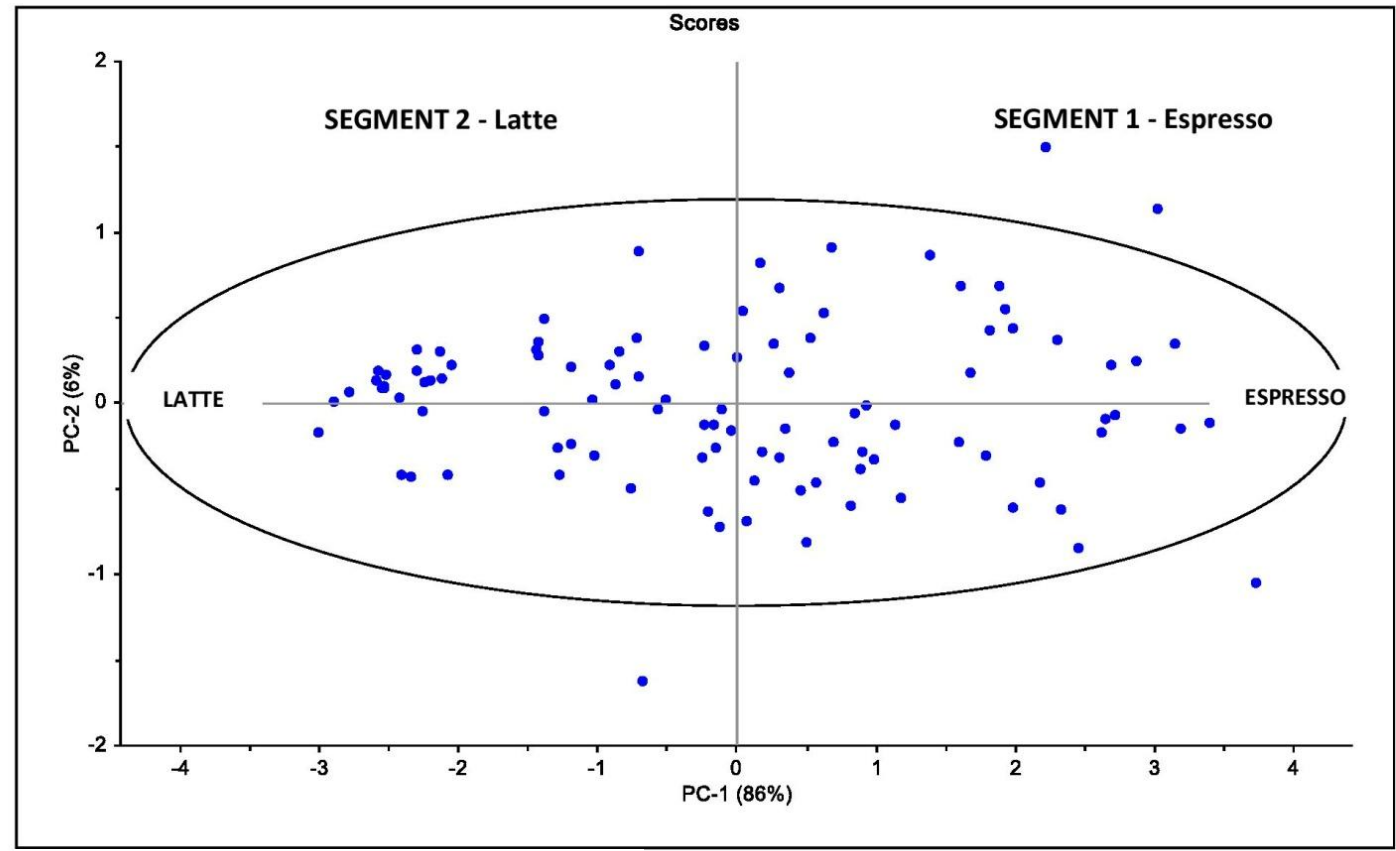

Figure 2 


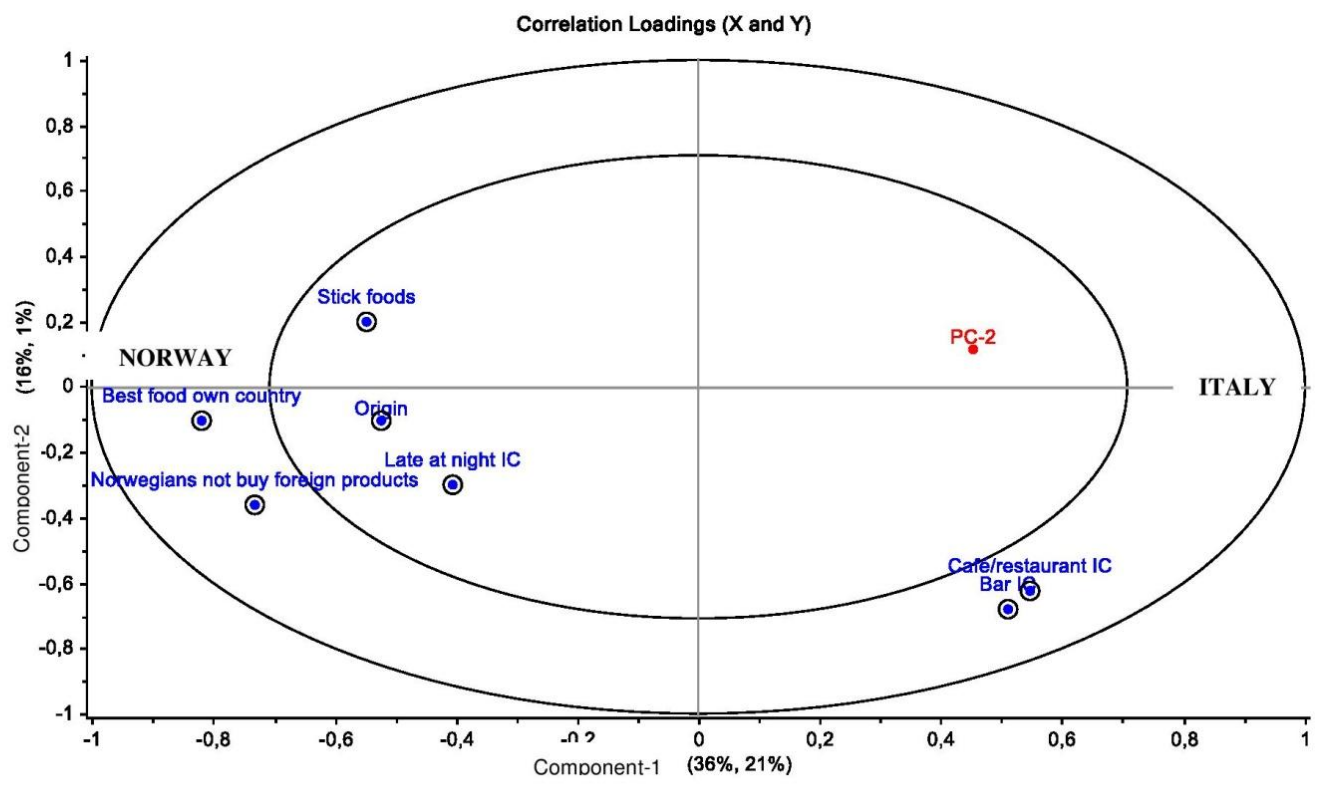

Figure 3 


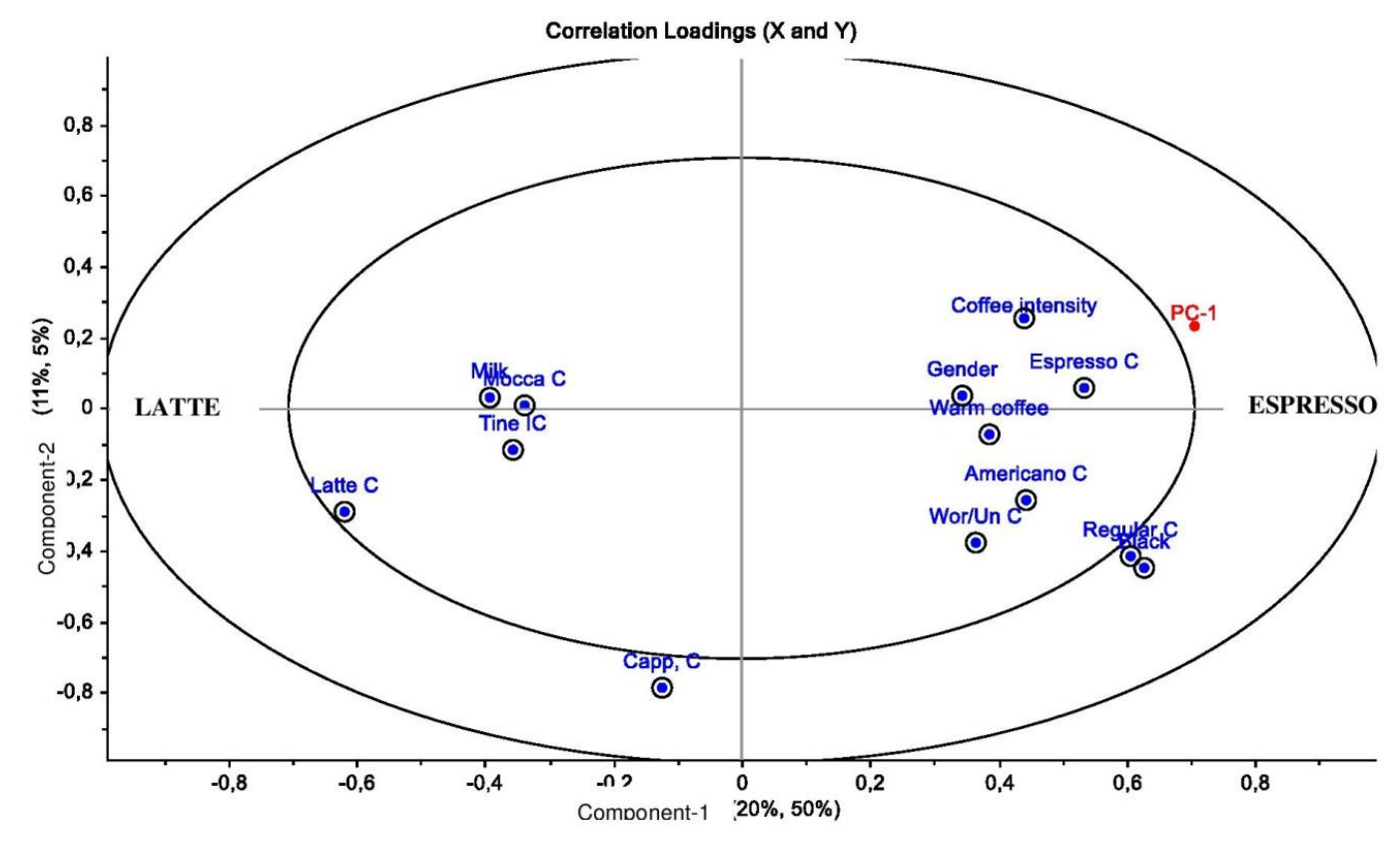

Figure 4 


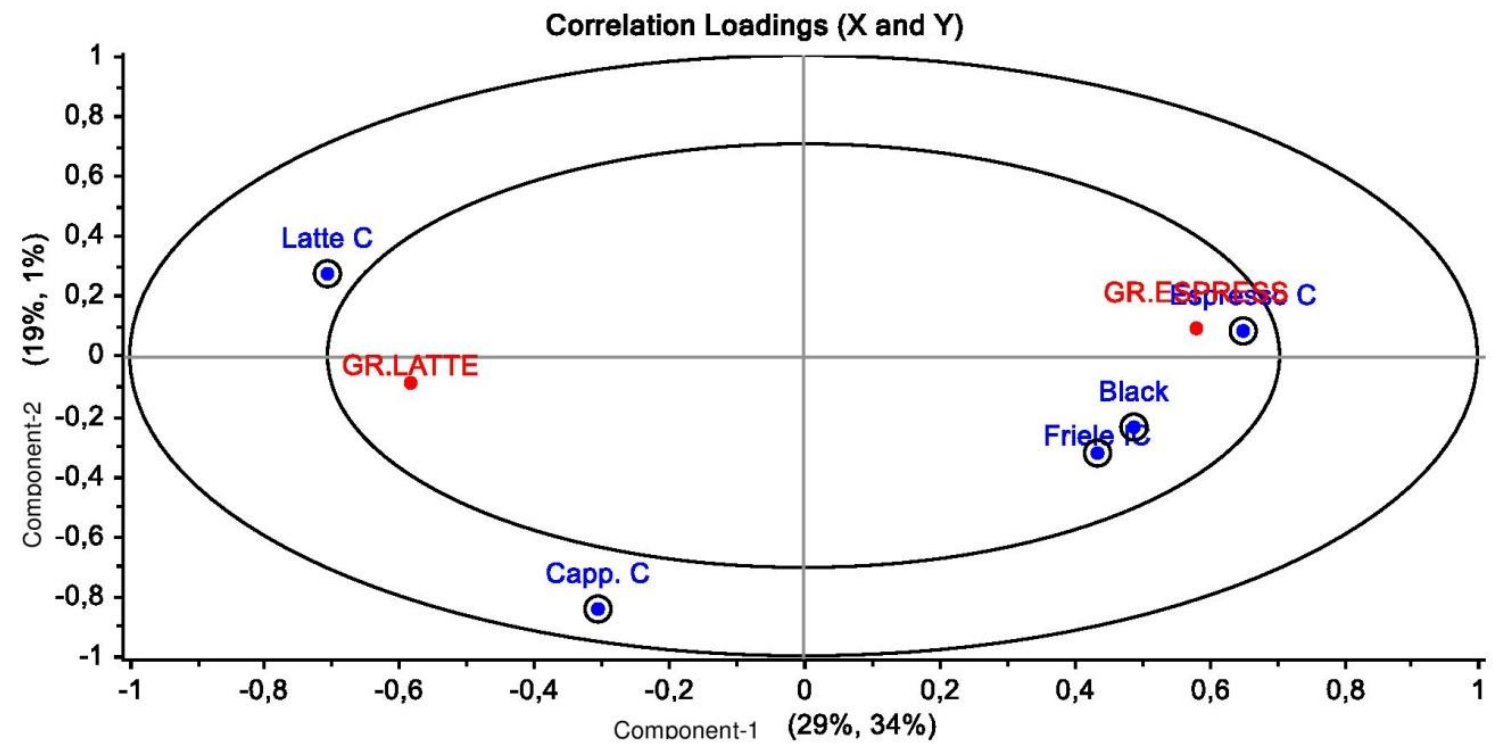

Figure 5 